\title{
CONHECIMENTO DA POPULAÇÃO SOBRE O ANESTESISTA E A ÁREA MÉDICA
}

SAÚDE COLETIVA/ EPIDEMIOLOGIA

CENTRO UNIVERSITÁRIO SÃO CAMILO

Silva, L.L. 1

Canga, L.A. ${ }^{1}$

Melaré, A.F. ${ }^{1}$

Oliveira, L.M de ${ }^{1}$

Alfano, A.M.V.L. ${ }^{2}$

1Acadêmicos de Medicina do Centro Universitário São Camilo;
${ }^{2}$ Docente de Anestesiologia do Centro Universitário São Camilo

Rua Tupiniquins, № 51 - Jardim Bandeirantes - Ribeirão Pires - SP

(11) 97654-9893 leandro.leal@aluno.saocamilo-sp.br 


\section{CONHECIMENTO DA POPULAÇÃO SOBRE O ANESTESISTA E A ÁREA MÉDICA}

SAÚDE COLETIVA/ EPIDEMIOLOGIA

\section{DESCRITORES}

Anestesia, anestesista, anestesiologia 


\title{
CONHECIMENTO DA POPULAÇÃO SOBRE O ANESTESISTA E A ÁREA MÉDICA
}

\author{
SILVA, L.L. ${ }^{1}$; CANGA, L.A. ${ }^{1}$; MELARÉ, A.F. ${ }^{1}$; OLIVEIRA, L.M de ${ }^{1}$; ALFANO, A.M.V.L. ${ }^{2}$ \\ ${ }^{1}$ Acadêmicos de Medicina do Centro Universitário São Camilo; \\ ${ }^{2}$ Docente de Anestesiologia do Centro Universitário São Camilo
}

\begin{abstract}
Resumo
Introdução: Mesmo a comunidade científica sabendo da importância do profissional anestesiologista, é sabido que diversos pacientes revelam desconhecimento acerca da formação e da função desse profissional.
\end{abstract}

Objetivos: Analisar tais conhecimentos de participantes de um ambulatório beneficente de Guarulhos - SP.

Método: Estudo descritivo em ambulatório na cidade de Guarulhos - SP, realizado de acordo com a Resolução 510 de 2016. Foi aplicado um questionário sobre o profissional anestesista. Os critérios de exclusão foram questionários incompletos e menores de 18 anos. Os dados foram analisados por frequência simples, porcentagem, teste exato de Ficher e teste do Qui Quadrado. Foi utilizado o software IBM SPSS Statistics 22 para a análise estatística e o Microsoft Excel 2016 para a organização dos dados.

Resultados: Foram analisados 96 questionários completamente preenchidos, sendo 63 (65,6\%) de mulheres e $33(34,4 \%)$ de homens; $51 \%$ dos indivíduos afirmaram que o profissional anestesista é médico. A maioria dos participantes não sabe quais são as atribuições do anestesista durante o procedimento cirúrgico, mesmo os que já foram submetidos a algum procedimento cirúrgico com anestesia. Apenas $13,5 \%$ dos participantes alegaram que o anestesista era o responsável por decidir o tipo de anestesia a ser usada na cirurgia enquanto que aproximadamente $70 \%$ dos indivíduos afirmaram que tal decisão era conjunta com o cirurgião. Em relação à nota média a ser dada ao anestesista e ao cirurgião quanto à importância dos mesmos na cirurgia foi de 9,65 (+-1,24) e $9,84(+-0,63)$ respectivamente.

Conclusão: Foi avaliado que o conhecimento da população pesquisada não foi satisfatório. Muitos entrevistados tiveram dúvidas no momento de responder, demostrando a falta de informação adequada ao público leigo sobre a atuação do profissional anestesista. Os resultados obtidos a partir dessa pesquisa vão de encontro com os dados encontrados na literatura, que também demostraram a necessidade de maior divulgação do conhecimento sobre a área anestésica para a população geral.

Palavras-chave: Anestesia, anestesista, anestesiologia. 


\section{INTRODUÇÃO}

Mesmo a comunidade científica sabendo da importância do profissional anestesiologista, é sabido que diversos pacientes revelam desconhecimento acerca da formação e da função desse profissional. ${ }^{1,2}$ Dessa forma, este presente trabalho tem como objetivo analisar tais conhecimentos de participantes de um ambulatório beneficente de Guarulhos - SP.

\section{MÉTODOS}

Foi realizado um estudo descritivo em ambulatório na cidade de Guarulhos - SP, durante um evento social desenvolvido no dia 12 de outubro de 2017. O evento era aberto ao público em geral, logo, foi abordado um público heterogênio tanto em questões de classe social quanto de acesso ao sistema de saúde (se público ou privado), contudo, tais fatores, propriamente ditos, não foram abordados neste presente trabalho, visto que o intuito era avaliar o conhecimento da população em geral.

Foi aplicado um questionário sobre o profissional anestesista. Tal questionário já foi utilizado em outros estudos sobre o mesmo tema em diferentes países com a mesma função, no entanto, os questionários eram apresentados intra-hospitalares, diferente do proposto no presente projeto.

O trabalho foi realizado de acordo com a RESOLUÇÃO № 510, DE 07 DE ABRIL DE 2016, a qual trata sobre as normas aplicáveis à pesquisa em Ciências Humanas e Sociais em trabalhos que lidam com dados obtidos diretamente do participante da pesquisa quanto a NÃO avaliação e registro pelo sistema COEP: ${ }^{3}$

"I - pesquisa de opinião pública com participantes não identificados;

IV - pesquisa censitária; e

VII - pesquisa que objetiva o aprofundamento teórico de situações que emergem espontânea e contingencialmente na prática profissional, desde que não revelem dados que possam identificar o sujeito"

O questionário foi aplicado pelos membros da Liga Acadêmica de Anestesiologia São Camilo, que foram previamente informados do modo de abordagem a fim de minimizar o viés das respostas pelos participantes da pesquisa.

Os participantes do evento, maiores de 18 anos e que concordassem em assinar o Termo de Consentimento Livre e Esclarecido (TCLE) responderam 10 (dez) perguntas, dentre os quais a 
idade, sexo, escolaridade, se já passou por algum procedimento cirúrgico submetido a algum efeito anestésico; que profissional é o anestesista; funções do anestesista e notas de 0 a 10 para o anestesista e cirurgião segundo a importância dos mesmos durante o procedimento.

Os critérios de exclusão foram questionários incompletos e menores de 18 anos. Os dados foram analisados por frequência simples, porcentagem, teste exato de Ficher e teste do Qui Quadrado. Foi utilizado o software IBM SPSS Statistics 22 para a análise estatística e Microsoft Excel 2016 para a organização dos dados.

\section{RESULTADOS}

Foram avaliados 96 questionários devidamente preenchidos, dentre os quais $63(65,6 \%)$ eram mulheres e 33 (34,4\%), homens entre 20 e 77 anos. (Quadro 1). Pode ser observado também que a maioria dos participantes cursaram até o ensino médio (39\%) e que $81 \%$ do total já foi submetido a um procedimento anestésico prévio, destes, 36,5\% em apenas um procedimento, enquanto que apenas $18,8 \%$ dos participantes não foram submetidos a nenhum procedimento anestésico (Gráfico $1)$.

Quadro 1 - Idade, Sexo, Escolaridade e experiência anestésica prévia dos indivíduos avaliados

\begin{tabular}{|c|c|c|c|c|c|c|c|c|}
\hline \multirow{2}{*}{ Idade } & \multirow{2}{*}{ № } & \multicolumn{2}{|c|}{ Sexo } & \multicolumn{3}{c|}{ Escolaridade } & \multicolumn{2}{c|}{$\begin{array}{c}\text { Procedimento } \\
\text { anestésico prévio }\end{array}$} \\
\cline { 3 - 9 } & & Feminino & Masculino & Fundamental & Médio & Superior & Sim & Não \\
\hline $20 \vdash 30$ & $18(19 \%)$ & 12 & 6 & 0 & 4 & 14 & 13 & 5 \\
\hline $30 \vdash 40$ & $12(13 \%)$ & 6 & 6 & 1 & 8 & 3 & 8 & 4 \\
\hline $40 \vdash 50$ & $29(30 \%)$ & 20 & 9 & 5 & 13 & 11 & 25 & 4 \\
\hline $50 \vdash 60$ & $25(26 \%)$ & 17 & 8 & 13 & 10 & 2 & 21 & 4 \\
\hline $60 \vdash 70$ & $8(8 \%)$ & 4 & 4 & 7 & 1 & 0 & 7 & 1 \\
\hline $70 \vdash 80$ & $4(4 \%)$ & 4 & 0 & 3 & 1 & 0 & 4 & 0 \\
\hline Total & 96 & $\mathbf{6 3}(\mathbf{6 6} \%)$ & $\mathbf{3 3 ( 3 4 \% )}$ & $\mathbf{2 9 ( 3 0 \% )}$ & $\mathbf{3 7}(\mathbf{3 9 \% )}$ & $\mathbf{3 0 ( 3 1 \% )}$ & $\mathbf{7 8 ( 8 1 \% )}$ & $\mathbf{1 8}(\mathbf{1 9} \%)$ \\
\hline
\end{tabular}

Gráfico 1: Já passou por algum procedimento cirúrgico submetido a algum efeito anestésico?




Em relação às perguntas referentes ao anestesista, $51 \%$ dos participantes informaram que 0 profissional é médico, enquanto que a segunda maior resposta foi que o profissional era técnico, com $28,1 \%$ das respostas, conforme pode ser observado no gráfico 2 .

Gráfico 2: Que profissional é o anestesista?

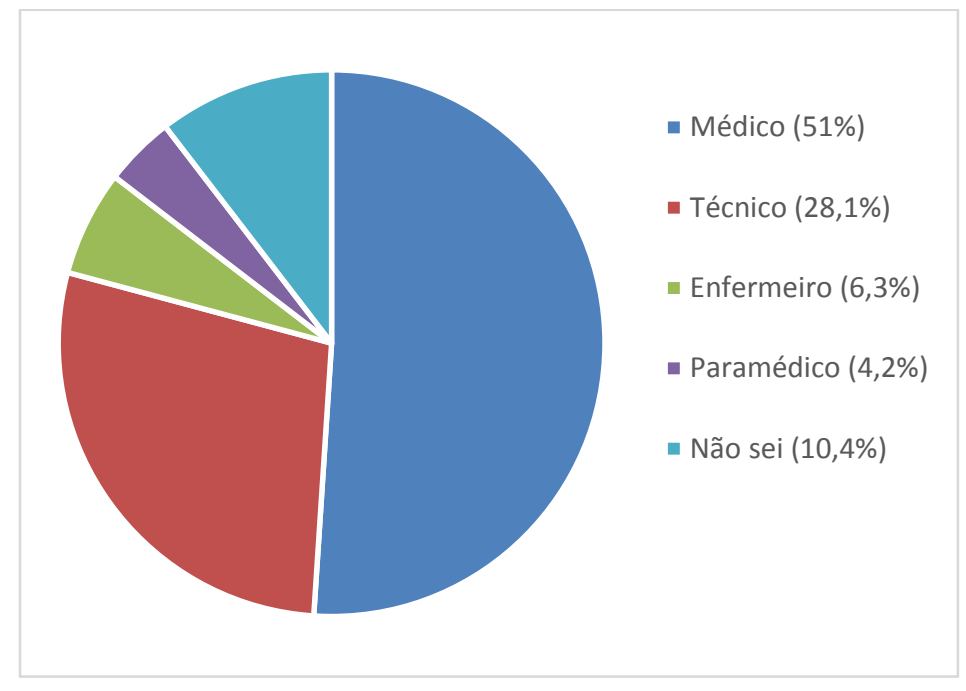

A maioria dos participantes alegou que o anestesista cuida do paciente durante o procedimento cirúrgico $(43,8 \%)$, enquanto que $25 \%$ afirmou que o profissional saía da sala para anestesiar outros pacientes em outras salas, conforme o gráfico 3.

Gráfico 3: O que o anestesista faz durante o procedimento cirúrgico?

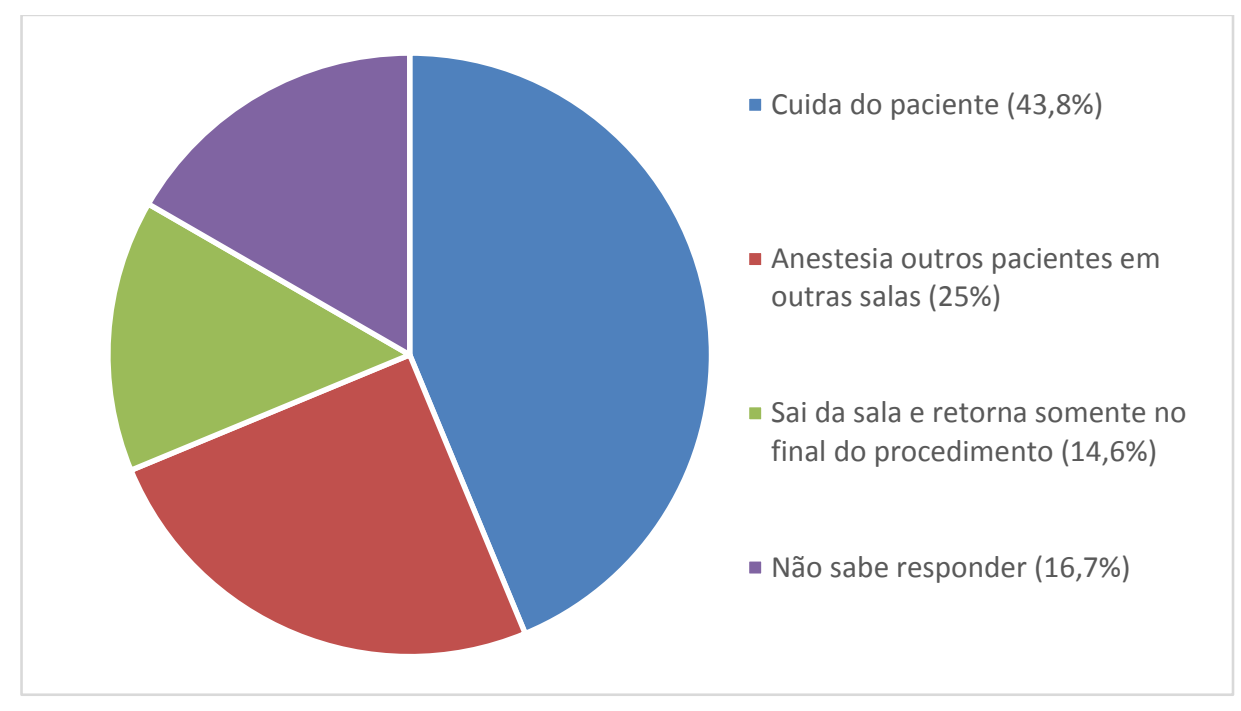

Em relação às atribuições do anestesista durante o procedimento cirúrgico, nenhum dos participantes marcou todas as respostas corretas (questão com múltiplas escolhas), logo, ou havia 
atribuição que não era do anestesista ou faltava atribuição que é deste. Os resultados para as funções que foram consideradas do anestesista podem ser observados na tabela 1.

\begin{tabular}{ccc}
\hline \multicolumn{3}{c}{ Tabela 1: É trabalho do anestesista: } \\
\hline $\begin{array}{c}\text { Dar pontos na pele no final da cirurgia } \\
\text { Fazer raio X durante a cirurgia }\end{array}$ & 11 & $11,57 \%$ \\
$\begin{array}{c}\text { Certificar-se de que o paciente acordou } \\
\text { sem problemas }\end{array}$ & 6 & $6,30 \%$ \\
$\begin{array}{c}\text { Dar instrumentos cirúrgicos ao cirurgião } \\
\text { Determinar se o paciente está apto a ser } \\
\quad \text { submetido ao procedimento }\end{array}$ & 52 & $54,20 \%$ \\
$\begin{array}{c}\text { Controlar a frequência cardíaca e a PA } \\
\text { Certificar-se de que o paciente não está } \\
\text { vomitando após a cirurgia }\end{array}$ & 12 & $12,50 \%$ \\
$\begin{array}{c}\text { Certificar-se de que o paciente não está } \\
\text { com dor após a cirurgia }\end{array}$ & 25 & $64,60 \%$ \\
$\quad$ Aplicar medicamentos e soro & 26 & $43,80 \%$ \\
Transfundir sangue se for necessário & $26 \%$ \\
$\quad$ Nenhuma das anteriores & 31 & $27,10 \%$ \\
\hline
\end{tabular}

Também foi questionado aos participantes quem era o profissional responsável por decidir o tipo de anestesia que seria utilizada no paciente durante o procedimento cirúrgico e apenas 5 indivíduos $(5,2 \%)$ deram o crédito ao anestesista, enquanto que a maioria das respostas foi de que a decisão é do cirurgião junto com o anestesista (67 indivíduos - 69,8\%), enquanto que ninguém afirmou que o paciente (uma das alternativas) poderia decidir o tipo de anestesia a ser utilizada (Gráfico 4).

Gráfico 4: Qual profissional é responsável por decidir o tipo de anestesia a ser usada em um procedimento cirúrgico?

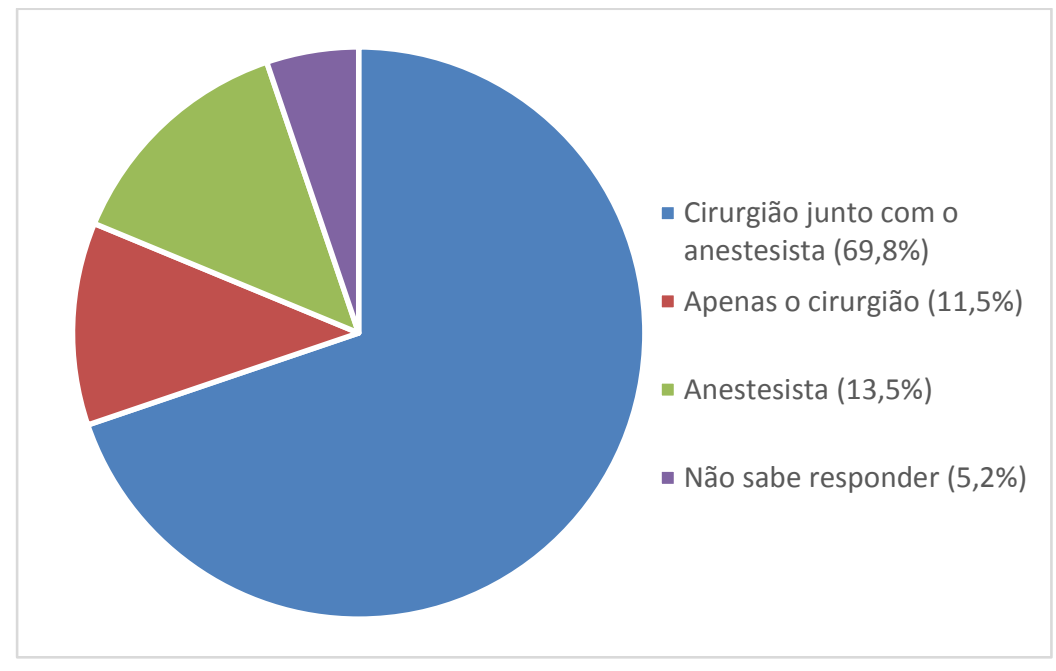


Foi solicitado, ainda, que os indivíduos avaliassem com nota de 0 a 10 referente a importância do anestesista para o procedimento cirúrgico (sendo 10 a importância máxima) e a mesma avaliação para o cirurgião, sendo a nota média de 9,65 (desvio padrão de 1,24) e 9,84 (desvio padrão de 0,63) respectivamente, logo, sem diferenças significativas.

\section{DISCUSSÃO}

Apesar do aumento da disponibilidade de informações e o contato do anestesiologista com o paciente, o conhecimento dos pacientes é limitado, pois a metade dos participantes não reconhecem o anestesiologista como médico, e estes dados estão de acordo com a literatura prévia, em que o reconhecimento do anestesiologista como médico especialista variou de $50 \%$ a 99\%. ${ }^{1,5,6,7}$. Além disso, a maioria dos participantes não sabe quais são as atribuições do anestesista durante o procedimento cirúrgico, mesmo os que já foram submetidos a algum procedimento cirúrgico com anestesia, em contraste com a literatura ${ }^{1,2}$ (Teste exato de Fisher é de 0,84 ( $\left.p>0,05\right)$ ) e de modo semelhante em relação à escolaridade (Qui-quadrado de $0,73(p>0,05)$ ). Conforme o Observado no Quadro 1:

Quadro 1: Experiência anestésica prévia e o

reconhecimento do profissional anestesista como

\begin{tabular}{|c|c|c|c|c|}
\hline \multicolumn{5}{|c|}{ médico $(\mathrm{em} \%)$} \\
\hline \multirow{2}{*}{\multicolumn{2}{|c|}{ Dados Analisados }} & \multicolumn{2}{|c|}{$\begin{array}{c}\text { Conhece o profissional } \\
\text { anestesista como } \\
\text { médico? }\end{array}$} & \multirow[t]{2}{*}{ Total } \\
\hline & & Sim & Não & \\
\hline \multirow{3}{*}{$\begin{array}{l}\text { Submetido a } \\
\text { dois ou mais } \\
\text { procedimentos } \\
\text { Total }\end{array}$} & Sim & 21,9 & 22,9 & 44,8 \\
\hline & Não & 29,2 & 26,0 & 55,2 \\
\hline & & 51,0 & 49,0 & 100,0 \\
\hline
\end{tabular}

O presente estudou mostrou que $51,0 \%$ dos participantes reconheceram o profissional como médico, sendo 29,2\% dessa quantia representada pelos indivíduos que não foram submetidos a dois ou mais procedimentos anestésicos, contra apenas $21,9 \%$. O que demonstra que ser submetido a mais de um procedimento não é um fator de importância para o reconhecimento do profissional. Em relação a não reconhecer o profissional como médico não houve diferença significativa entre ser submetido a mais de um procedimento anestésico. Como o resultado do Teste exato de Fisher é de 0,84 ( $p>0,05)$, NÃO existe predileção do paciente ser submetido a dois ou mais procedimentos e reconhecer o profissional anestesista como médico, pois, o p obtido é insignificante. A mesma correlação foi encontrada em um estudo israelense que também não mostrou diferença entre o grupo que já fora anestesiado e o grupo que se encontrava em sua primeira experiência anestésica. ${ }^{4}$ Entretanto, esse dado diverge de outros estudos, que mostraram 
correlação significativa entre os pacientes bem informados sobre a qualificação médica do anestesiologista e a presença de experiência anestésica prévia. ${ }^{1,2}$

Este estudo não demonstrou correlação significativa entre o reconhecimento da profissão do Anestesista e a escolaridade do indivíduo, enquanto que na literatura a escolaridade foi um fator significante ${ }^{1,8}$. Dos participantes que reconheceram o anestesiologista como médico (51,0\%), 18,8\% tinham o ensino superior, enquanto que $21,9 \%$ tinham o ensino médio. Já os $28,1 \%$ do total de parcitipantes que afirmaram que o anestesista era técnico, 7,3\% apresentava ensino superior, semelhante ao no dos que tem apenas o ensino fundamental. Como o resultado de Qui-quadrado é de $0,87(p>0,05)$ podemos afirmar que NÃO há relação entre a idade e o reconhecimento do anestesista como médico.

Quadro 2: Escolaridade e o conhecimento sobre a profissão do Anestesista (em \%)

\begin{tabular}{|c|c|c|c|c|c|c|c|}
\hline \multirow{2}{*}{\multicolumn{2}{|c|}{ Dados Analisados }} & \multicolumn{5}{|c|}{ Qual é a profissão do Anestesista? } & \multirow[b]{2}{*}{ Total } \\
\hline & & Médico & Técnico & Enfermeiro & Paramédico & $\begin{array}{l}\text { Não sabe } \\
\text { responder }\end{array}$ & \\
\hline \multirow{4}{*}{  } & Fundamental & 10,4 & 7,3 & 0,0 & 2,1 & 6,3 & 26,0 \\
\hline & Médio & 21,9 & 13,5 & 2,1 & 2,1 & 1,0 & 40,6 \\
\hline & Superior & 18,8 & 7,3 & 4,2 & 0,0 & 3,1 & 33,3 \\
\hline & Total & 51,0 & 28,1 & 6,3 & 4,2 & 10,4 & 100,0 \\
\hline
\end{tabular}

No presente estudo $13,5 \%$ dos participantes alegaram que o anestesista era o responsável exclusivo por decidir o tipo de anestesia a ser usada na cirurgia. Enquanto que aproximadamente $70 \%$ alegaram que essa era ação conjunta entre o anestesista e o cirurgião. Apesar disso, a nota média dada ao anestesista e ao cirurgião quanto a importância dos mesmos na cirurgia foi de 9,65 $(-1,24)$ e $9,84(-0,63)$ respectivamente, mesmo que $49 \%$ dos indivíduos pesquisados desconhecem a formação médica do anestesiologista. E estes dados estão de acordo com a literatura prévia1.

Alguns estudos ${ }^{4,8,9}$ propõem que o anestesiologista deve se apresentar de modo eficaz ao paciente, principalmente na visita pré-anestésica, onde deve-se estabelecer a relação médico paciente, esclarecer sobre o procedimento a ser realizado e alcançar o reconhecimento profissional dos pacientes, pois muitos dos indivíduos questionados no presente trabalho não atribuíram ao anestesiologista papel fundamental no cuidado perioperatório, como, por exemplo, determinar se o paciente está apto a ser submetido ao procedimento, controlar a frequência cardíaca e a PA, realização de transfusão, certificar que o paciente acordou sem problemas da cirurgia, tratamento da dor e náuseas no pós operatório, o que mostra a necessidade de esclarecer para a população o que é a anestesiologia e quais as áreas específicas em que o anestesiologista pode intervir para que a visão do profissional pelo doente não continue sendo subvalorizada. 


\section{CONCLUSÃO}

Foi avaliado que o conhecimento da população pesquisada não foi satisfatório. Muitos entrevistados tiveram dúvidas no momento de responder, demostrando a falta de informação adequada ao público leigo sobre a atuação do profissional anestesista. Os resultados obtidos a partir dessa pesquisa vão de encontro com os dados encontrados na literatura: há a necessidade de maior divulgação do conhecimento sobre a área anestésica para a população geral.

\section{REFERÊNCIAS}

1. Oliveira, Katia Ferreira de; Clivatti, Jefferson; Munechika, Masashi; et al. O que o paciente sabe sobre o trabalho do anestesiologista?. Rev. Bras. Anestesiol. 2011, vol.61, n.6. pp.724-727.

2. Bagabas, Ahmed M.; Kashi, Mooataz Mohammed; Alamoudi, Ahmed O.; et al. Knowledge about anesthesia and the role of anesthesiologists among Jeddah citizens. International Journal of Research in Medical Sciences, [S.I.], v. 5, n. 6, p. 2779-2783. may 2017. ISSN 2320-6012.

3. Guerriero lara Coelho Zito. Approval of the Resolution governing the ethics of research in social sciences, the humanities, and other disciplines that use methodologies characteristic of these areas: challenges and achievements. Ciênc. saúde coletiva [Internet]. 2016 Aug [cited 2018 Aug 04] ; 21 ( 8 ): 2619-2629. Available from: http://www.scielo.br/scielo.php?script=sci_arttext\&pid=S141381232016000802619\&lng=en. http://dx.doi.org/10.1590/1413-81232015218.17212016.

4. Calman LM, Mihalache A, Evron S et al. - Current understanding of the patient's attitude toward the anesthetist's role and practice in Israel: effect of the patient's experience. J Clin Anesth, 2003;15:451- 454

5. Ribeiro CS, Mourão JIB. O anestesiologista: a visão do doente. Rev Bras Anestesiol. 2015; 65(6): 497-503.

6. Gottschalk A, Seelen S, Tivey S, et al. What do patients know about anesthesiologists? Results of a comparative survey in an U.S., Australian, and German university hospital. J Clin Anesth. 2013;25:85---91.

7. Leite F, da Silva LM, Biancolin SE, et al. Patient perceptions about anesthesia and anesthesiologists before and after surgical procedures. Sao Paulo Med J. 2011;129:224---9.

8. Hariharan S, Merritt-Charles L, Chen D - Patient perception of the role of anesthesiologists: a perspective from the Caribbean. J Clin Anesth, 2006;18:504-509.

9. Lopes CA, Machado PRA, Castiglia YMM - O que pensa o paciente sobre o binômio anestesiologista-anestesia. Rev Bras Anestesiol, 1993;43(5):335-340. 